\title{
Non-medical use of opioids among HIV-infected opioid dependent individuals on opioid maintenance treatment: the need for a more comprehensive approach
}

Perrine Roux ${ }^{1,2,3^{*}}$, Patrizia M Carrieri ${ }^{1,2,3}$, Julien Cohen ${ }^{1,2,3}$, Isabelle Ravaux ${ }^{4}$, Bruno Spire ${ }^{1,2,3}$, Michael Gossop ${ }^{5}$ and Sandra D Comer ${ }^{6}$

\begin{abstract}
Background: Opioid maintenance treatment (OMT) has a positive impact on substance use and health outcomes among HIV-infected opioid dependent patients. The present study investigates non-medical use of opioids by HIVinfected opioid-dependent individuals treated with buprenorphine or methadone.

Methods: The MANIF 2000 study is a longitudinal study that enrolled a cohort of 476 HIV-infected opioid-dependent individuals. Data were collected in outpatient hospital services delivering HIV care in France. The sample comprised all patients receiving OMT (either methadone or buprenorphine) who attended at least one follow-up visit with data on adherence to OMT ( $\mathrm{N}=235$ patients, 1056 visits). Non-medical use of opioids during OMT was defined as having reported use of opioids in a non-medical context, and/or the misuse of the prescribed oral OMT by an inappropriate route of administration (injection or sniffing). After adjusting for the non-random assignment of OMT type, a model based on GEE was then used to identify predictors of non-medical use of opioids.

Results: Among the 235 patients, 144 (61.3\%) and 91 (38.9\%) patients were receiving buprenorphine and methadone, respectively, at baseline. Non-medical use of opioids was found in $41.6 \%$ of visits for $83 \%$ of individual patients. In the multivariate analysis, predictors of non-medical use of opioids were: cocaine, daily cannabis, and benzodiazepine use, experience of opioid withdrawal symptoms, and less time since OMT initiation.

Conclusions: Non-medical use of opioids was found to be comparable in OMT patients receiving methadone or buprenorphine. The presence of opioid withdrawal symptoms was a determinant of non-medical use of opioids and may serve as a clinical indicator of inadequate dosage, medication, or type of follow-up. Sustainability and continuity of care with adequate monitoring of withdrawal symptoms and polydrug use may contribute to reduced harms from ongoing non-medical use of opioids.
\end{abstract}

Keywords: opioid maintenance treatment, buprenorphine, methadone, non-medical use, HIV, withdrawal, antiretrovirals

\section{Background}

Among HIV-infected opioid dependent individuals, the clinical management of drug dependence is a matter of great concern. This issue is especially relevant in those countries where the HIV epidemic is driven by injecting drug users (IDUs) [1,2]. Even in industrialized countries,

\footnotetext{
* Correspondence: perrine.roux@inserm.fr

'INSERM, U912 (SE4S), 23 rue Stanislas Torrents, 13006 Marseille, France Full list of author information is available at the end of the article
}

HIV-infected opioid-dependent persons seeking care for their drug dependence may face many barriers to effective treatment, and their management may be complicated by the difficulties associated with the provision of multiple treatments [3]. Opioid maintenance treatment (OMT) has been found to reduce high risk behaviors related to HIV transmission such as injecting drugs, sharing needles/syringes, and having unprotected sex [4]. In France, two forms of OMT, with buprenorphine 
and methadone, are available and provision of these treatments has been found to have a substantial beneficial impact upon the growth of the HIV epidemic [5].

The initiation of OMT with methadone or buprenorphine in HIV-infected opioid dependent patients has been found to have a positive impact on health outcomes [6], and plays an important role in sustaining adherence to antiretroviral treatment (ART) for HIV infection [7]. The topic of adherence to ART has been widely studied since the beginning of the HIV epidemic [8]. In injecting drug users (IDUs) [9], adherence to ART is important because sub-optimal adherence to ART may lead to the risk of HIV resistance and accelerated progression of disease [10]. In this paper, we focused on non-medical use of opioids defined as either use of illicit opioids such as heroin or other non-prescribed opioids, or use of OMT (buprenorphine or methadone) by a non medically prescribed route of administration. Non-medical use of opioids, especially by injection, is particularly relevant in HIV-positive patients because it is a major correlate not only of response to OMT but also to antiretroviral therapy response as expressed by non-adherence [7] and virological failure [11].

The MANIF 2000 cohort study took place in several settings in France and was designed to focus on sociobehavioral aspects of HIV-positive IDUs, with particular emphasis on their access and adherence to antiretroviral treatment as well as OMT-related outcomes. The inclusion of HIV-infected opioid-dependent individuals, while buprenorphine and methadone were launched to treat opioid drug dependence, provided us with the opportunity to identify the correlates of non-medical use of opioids during opioid maintenance treatment.

\section{Methods}

\section{Cohort and sample}

The French MANIF 2000 cohort in 1995/1996 enrolled 467 patients who were HIV-positive. Inclusion criteria for enrollment in the cohort were: receiving OMT treatment, patients with a CD4+ cell count $>300$ during the last visit prior to enrolment and in clinical stage A or B. This cohort was designed to focus on social and behavioral aspects of HIV-positive IDUs and particularly on their access [12] and adherence to antiretroviral treatment [13] as well as to OMT [14]. In this study, we only selected visits during time periods when patients were enrolled in OMT, either methadone or buprenorphine, and had available data on opioid use, including OMT. The sample comprised 235 individuals, accounting for a total of 1056 visits. All individuals who agreed to be interviewed signed an informed consent form, approved with the study protocol by the Committee for the Protection of Persons (CPP) involved in biomedical research.

\section{Data collection}

Data were collected at 6-month intervals by means of a face-to-face interview, a self-administered questionnaire, and medical records. The face-to-face interview was based on a standardized protocol, administered by trained nurses, which gathered psychosocial information and patients' personal experience with HIV infection and care. The self-administered questionnaire included socio-demographic data, prison history, substance use and related behaviours, OMT exposure (methadone or buprenorphine), and consumption of psychotropic drugs and alcohol during the previous 6 months. Self-reported use of heroin and morphine were checked for validity at enrollment by morphine detection in urine samples. Injection drug use at any given visit thereafter was defined as the injection of any drug in the 6 months before that visit.

Clinical and laboratory data on viral load, CD4 T-cell counts and data on HIV clinical stage, with stage $\mathrm{C}$ indicating progression to AIDS disease were collected every 6 months from the physician or from medical records [15]. An undetectable viral load was defined as an HIV1 RNA level below the lower limit of detection of the assay and was considered a virological success. Information was also collected about the first positive HIV test and patients' ART history.

Depression was measured using the French version of the Center for Epidemiological Studies Depression Scale (CES-D) [16]. The 75th percentile (age-gender specific) of the distribution of values for the corresponding indicator in the general French population was used to classify patients as depressed or not depressed at each interview. Then, the CES-D score was dichotomized by using 17 and 23 as cut-off points for men and women, respectively, as indicative of depression, on a score scale ranging from 0 to 60 , as already validated in a previous study [17].

Involvement with "non-medical use of opioids" was collected through both patients' and physicians' answers to the questionnaires. There was little discrepancy between physicians' and patients' reports about OMT: in cases of disagreement, patients' self reports were considered as more reliable and were used in the analyses. At any given visit, time since OMT initiation was computed as the uninterrupted time-interval between the last initiation or re-initiation of OMT until that visit. Also, the prescribed dose of OMT was considered low if the methadone dose was less than $60 \mathrm{mg}$ per day or the buprenorphine dose was less than $8 \mathrm{mg}$ per day.

Information about the use of opioids and OMT was collected by means of the self-administered questionnaire and the structured face-to-face interview. Nonmedical use of opioids was defined as use of illicit or non-prescribed opioids, and/or the misuse of the 
prescribed OMT by using an inappropriate route of drug administration. Specifically, individuals were considered as having non-medical use of opioids if at any given visit they reported use of any opioid drug other than their prescribed OMT medication, or if they reported having administered their prescribed OMT by sniffing or injection in the previous 6 months.

\section{Statistical analysis}

A 2-step Heckman approach allowed us to account for the non-random assignment to different types of OMT medication. The first step, explained in a previous paper [18], was based on a probit model to identify predictors of starting either buprenorphine or methadone treatment and led to the computation of the inverse Mills ratio (IMR). The IMR was then introduced in the second step model to correct for the potential non-random assignment of buprenorphine and methadone (prescription bias).

A model based on Generalized Estimating Equations (GEE) was then used to identify predictors of non-medical use of opioids while correcting for the bias induced by non-random assignment. Variables with p-values < 0.20 in the univariate analysis of each step were considered eligible for inclusion in the multivariate models. An exchangeable correlation matrix was used for the GEE models. All variables tested, including the IMR, were considered eligible to enter the model. A backward procedure was used to identify the best GEE model and variables were removed one at a time based on a $p$ value $>0.05$. The log-likelihood ratio test was used to identify the best pattern of predictors. Bias-corrected confidence intervals and p-values were based on 400 bootstrap replications.

\section{Results}

\section{Descriptive results at baseline}

Among the 235 patients, 163 (69.4\%) were men and $72(30.6 \%)$ were women. The median [IQR] age was 34 [31-37] years. Thirty-four (14.8\%) patients had a high school certificate and $150(63.8 \%)$ were the owner or renter of their accommodation. Depressive symptoms were found in $170(73 \%)$ patients. Use of cocaine was reported by $85(36.5 \%)$ and heroin use by 141 (60.5\%). One hundred and forty eight (63\%) patients reported having injected any drug in the 6 months before the first visit on OMT. Heavy drinking was reported by 57 (24.9\%) patients. Buprenorphine was the more commonly prescribed type of medication: this was prescribed to $144(61.3 \%)$ patients at baseline, with 91 (38.9\%) receiving methadone. About one fifth of the patients $(\mathrm{n}=46,19.6 \%)$ were being treated with ART.

\section{Descriptive results during the study period}

The descriptive analysis of the whole study period is presented in Table 1.

During the study period, the median [IQR] time since the initiation of opioid maintenance treatment was 16 [13-20] months. Among the 235 patients, 18 switched from buprenorphine to methadone, 10 switched from methadone to buprenorphine, and one switched from buprenorphine to methadone and then back to buprenorphine. Among all the treatment visits, non-medical use of opioids was found in 439 (41.6\%) visits for 196 individual patients. In addition, the mean duration of study follow-up for our sample was 45 months.

\section{First step: Predictors of OMT prescription, buprenorphine or methadone}

In the first step model, factors independently associated with methadone prescription were unemployment, drinking more than 4 units of alcohol per day, cocaine use in the 6 months prior to the visit, and smoking more than 20 cigarettes per day [18].

\section{Second step: Univariate and multivariate analyses}

The results of the univariate analysis are presented in Table 1 . No statistically significant difference was found between type of maintenance medication (buprenorphine or methadone) with regard to non-medical use of opioids, after correcting for the bias induced by nonrandom assignment. Regarding HIV status, a number of variables were found to be associated with non-medical use of opioids in the univariate but not in the multivariate analyses: not receiving ART, being at clinical stage A (compared to $B$ and $C$ ), having a higher duration since first testing positive for HIV, not having an undetectable viral charge and not reporting having a complete trust in the physician. Socio-demographic factors that were found to be associated with non-medical use of opioids included younger age, and not being owner or renter of his/her accommodation. Patients who reported depressive symptoms were more likely to have reported nonmedical use of opioids. Lower doses of OMT were not significantly associated with non-medical use of opioids.

In the multivariate analysis presented in Table 2 five variables that were found to be significant in the univariate analysis remained significantly associated with the outcome. These included non-opioid drug use (use of cocaine and benzodiazepines, and daily use of cannabis). Patients who reported having experienced opioid withdrawal symptoms in the 6 months prior to the visit were found to report more non-medical use of opioids. Uninterrupted attendance at an OMT program until a given visit was associated with less non-medical use of opioids. 
Table 1 Factors associated with non-medical use of opioids during opioid maintenance treatment: univariate analyses.

\begin{tabular}{|c|c|c|c|c|}
\hline & $\begin{array}{l}\text { Number of visits } \\
(\%) \text { or median [IQR] }\end{array}$ & Nb. of patients & $\begin{array}{c}\text { Coefficient } \\
(95 \% \mathrm{Cl})\end{array}$ & p-value \\
\hline Buprenorphine (ref) & $640(60.6)$ & 154 & & \\
\hline Methadone & $416(39.4)$ & 111 & $0.00(-0.41 ; 0.37)$ & 0.98 \\
\hline Female gender & $286(27.1)$ & 72 & $0.16(-0.09 ; 0.39)$ & 0.20 \\
\hline $\mathrm{Age}^{\mathrm{a}}$ & 36 [32-39] & & $-0.69(-0.96 ;-0.43)$ & $<10^{-3}$ \\
\hline Owner or renter of their house & $757(71.7)$ & 189 & $-0.24(-0.45 ; 0.00)$ & 0.03 \\
\hline Good housing conditions $^{\mathrm{b}}$ & $868(82.4)$ & 210 & $-0.13(-0.39 ; 0.08)$ & 0.22 \\
\hline Heavy drinking ${ }^{c}$ & $213(20.4)$ & 95 & $0.15(-0.07 ; 0.40)$ & 0.20 \\
\hline Cocaine use $^{d}$ & $277(26.3)$ & 115 & $0.91(0.67 ; 1.20)$ & $<10^{-3}$ \\
\hline Cannabis use (daily) $^{d}$ & $467(44.4)$ & 156 & $0.45(0.29 ; 0.62)$ & $<10^{-3}$ \\
\hline Benzodiazepine consumption $^{d}$ & $358(33.9)$ & 150 & $0.62(0.44 ; 0.81)$ & $<10^{-3}$ \\
\hline \multicolumn{5}{|l|}{ HIV clinical stage } \\
\hline A (ref) & $474(44.9)$ & 138 & & \\
\hline B & $518(49.1)$ & 125 & $-0.24(-0.49 ;-0.01)$ & 0.06 \\
\hline C & $63(6.0)$ & 22 & $-0.68(-1.17 ;-0.28)$ & 0.003 \\
\hline Depressive symptoms $^{e}$ & $666(63.2)$ & 200 & $0.34(0.18 ; 0.52)$ & $<10^{-3}$ \\
\hline Withdrawal symptoms & $204(19.3)$ & 122 & $0.81(0.56 ; 1.07)$ & $<10^{-3}$ \\
\hline Time since first injection (years) ${ }^{a}$ & $16[13-20]$ & & $-0.56(-0.86 ;-0.32)$ & $<10^{-3}$ \\
\hline Time since first positive HIV test (years) ${ }^{a}$ & $11[8-13]$ & & $-1.00(-1.35 ;-0.76)$ & $<10^{-3}$ \\
\hline Time since OMT initiation (months) ${ }^{f}$ & $16[7-30]$ & & $-0.29(-0.39 ;-0.22)$ & $<10^{-3}$ \\
\hline Undetectable viral charge & $366(34.9)$ & 124 & $-0.25(-0.46 ;-0.03)$ & 0.03 \\
\hline CD4 cell count $/ \mathrm{mm}^{3}<200$ & $57(5.5)$ & 32 & $-0.26(-0.65 ; 0.15)$ & 0.20 \\
\hline Receiving ART & $395(37.4)$ & 119 & $-0.52(-0.70 ;-0.30)$ & $<10^{-3}$ \\
\hline Complete trust in physicians & $824(78.4)$ & 196 & $-0.33(-0.62 ; 0.10)$ & 0.01 \\
\hline Low prescribed dose of $\mathrm{OMT}^{\mathrm{g}}$ & $676(66.8)$ & 169 & $-0.22(-0.43 ; 0.04)$ & 0.06 \\
\hline
\end{tabular}

*MANIF 2000 cohort, $\mathrm{N}=235,1056$ visits.

${ }^{\text {a }}$ For ten years increase.

${ }^{b}$ Good housing conditions were defined as the rank 3 and 4 (quite or very comfortable vs. uncomfortable or low comfort) using a four-point Likert scale.

c Heavy drinking was defined as drinking more than 4 alcohol units on any one occasion.

${ }^{d}$ During the previous 6 months.

e Patients were defined with depressive symptoms if CES-D > 17 for men and $>23$ for women.

${ }^{f}$ For one year increase.

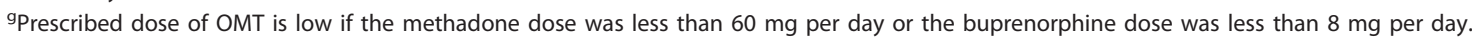

\section{Discussion}

Non-medical use of opioids was found to be comparable in HIV-infected opioid dependent patients receiving methadone or buprenorphine. In addition, our results showed that ongoing use of non-opioids (such as

Table 2 Factors associated with non-medical use of opioids during opioid maintenance treatment: multivariate analysis.

\begin{tabular}{lcc}
\hline & $\begin{array}{c}\text { Adjusted coefficient } \\
(\mathbf{9 5 \%} \mathrm{Cl})\end{array}$ & p-value \\
\hline Cocaine use $^{\mathrm{d}}$ & $0.81(0.57 ; 1.10)$ & $<10^{-3}$ \\
Cannabis use (daily) $^{\mathrm{d}}$ & $0.28(0.08 ; 0.47)$ & 0.01 \\
Benzodiazepines consumption $^{\mathrm{d}}$ & $0.37(0.16 ; 0.55)$ & $<10^{-3}$ \\
Withdrawal symptoms $^{-3}$ & $0.62(0.36 ; 0.89)$ & $<10^{-3}$ \\
Time since OMT initiation (months) $^{\mathrm{f}}$ & $-0.25(-0.35 ;-0.18)$ & $<10^{-3}$ \\
\hline
\end{tabular}

${ }^{\mathrm{d}}$ During the previous 6 months.

${ }^{f}$ For one year increase. cocaine, cannabis, and benzodiazepines), perception of withdrawal symptoms, and a shorter retention in OMT are associated with non-medical use of opioids. At a time when non-medical use of prescription opioids [19] and use of opioids by injection [20-22] are growing problems and a real concern for public health, identifying correlates of non-medical use of opioids is of interest and should help physicians to better manage opioid dependence and improve the effectiveness of OMT in not only HIV-infected population but also the whole population of opioid-dependent individuals.

First, it is interesting to note that, according to the results of the univariate analysis, HIV care has a positive impact on decreasing non-medical use of opioids. Patients who were receiving antiretroviral treatment (ART) were less likely to report non-medical use of opioids. This result may be related to the wider access to ART in IDUs who achieved stabilization on OMT 
[23]. Moreover, it is known that access to ART has a positive impact on drug injecting cessation in HIVinfected IDUs [24]. This impact could be explained by the reality of the relationship between physician and patient based upon trust in the ART initiation process [25], as suggested in our univariate analysis. This latter result supports the idea that access to ART in HIVinfected opioid dependent individuals could play a harm reduction role.

However, the results of the multivariate analysis show the most important predictors of non-medical use of opioids which are not related to HIV care in our analysis. And after multiple adjustments (including for non random assignment of buprenorphine and methadone), only shorter time since OMT initiation, experience of withdrawal symptoms, and non-opioid drug use were found to be significant determinants of non-medical use of opioids. Among the independent predictors of non-medical use of opioids, some patients' illicit substance use behaviors such as cocaine, daily cannabis, and benzodiazepine use were associated with the outcome. These findings are consistent with those of previous studies showing that non-opioid drug use in OMT-treated patients may negatively influence treatment outcomes. For instance, cocaine use during OMT has previously been shown to be a predictor of buprenorphine diversion by injection in patients receiving prescribed buprenorphine [14] and to hinder efficacy of OMT [26]. In addition, OMT patients who had used benzodiazepines were more likely to have opioid-positive urine screens during OMT [27]. This is a concern because few guidelines are available to physicians who are required to treat co-dependent drug users. A recent meta-analysis has summarized the most common approaches to treating benzodiazepine dependence [28] and the most effective is a combination of a gradual dose reduction regimen and psychological interventions. Moreover, a recent study showed that opioid dependent individuals receiving heroin-assisted treatment were more likely to decrease their benzodiazepine use compared to those receiving methadone maintenance treatment [29]. Finally, heavy cannabis use also has been found to be correlated with buprenorphine injection [30]. Polydrug use should be considered more thoroughly not only for measuring the independent effects of multiple drug use but also for investigating the effects of drug use patterns on behavioural outcomes [31].

Although the association between time since OMT initiation and non-medical use of opioids is not unexpected, it demonstrates that the longer the duration of OMT (retention in OMT), the better achievement of stabilization in terms of heroin use and OMT diversion [32]. The clinical management of opioid dependence has already been described as a very long process with a high risk of cycling in and out of treatment [33].
Patients who reported withdrawal symptoms during the study period were also found to report higher nonmedical use of opioids. This result is clinically very relevant and suggests that physicians should investigate subjective and objective withdrawal symptoms during treatment in order to better understand the reasons that inter-dose opioid withdrawal exist [34]. Their occurrence may be indicative of inadequate OMT dosage $[35,36]$, either too low, which leads to withdrawal symptoms directly, or too high, which leads to aversive side effects that lead to discontinuation of OMT. However, the results do not support the notion that lower doses of OMT are related to more non-medical use of opioids, probably because low doses were a proxy for less severe opioid dependence and not for inadequate dose prescription. Other factors could be related to disliking of the subjective effects of OMT, or polydrug use, which also may lead patients to interrupt treatment $[37,38]$. This issue is all more relevant since Mateu-Gelabert et al showed that withdrawal symptoms could increase risky behaviors regarding HIV and also Hepatitis $\mathrm{C}$ virus (HCV) transmission by undermining IDUs' willingness to inject safely [39].

Recent WHO guidelines recommend that the dose of OMT should be increased progressively according to clinical effect in the individual patient, up to an adequate dosage [40]. In addition, drug interactions represent a potential concern in HIV-infected individuals, especially with regard to methadone and protease inhibitors, and in these circumstances, methadone dose adjustments may be required to avoid withdrawal symptoms [41]. In addition, the experience of withdrawal symptoms among HIV-infected patients who are receiving OMT needs careful surveillance and clinical management since the presence of withdrawal symptoms has been associated with an increased risk of mortality in the same cohort [42].

After adjustment for non-random allocation to buprenorphine/methadone prescription, we found no difference between methadone and buprenorphine with regard to non-medical use of opioids. These findings are consistent with previous studies showing that methadone and buprenorphine are broadly equivalent at higher dosages [43]. The correction for the bias elicited by non-random assignment was necessary due to the two different models of OMT delivery in France which may lead to the selection of different opioid drug dependent populations. Historically, in France, initiation of methadone is possible only in specialized centers for drug dependence while access to buprenorphine is available in primary care settings [5]. This differential access could explain why methadone in France is more frequently initiated in patients presenting a severe addictive profile. In France, methadone programs provide 
multidisciplinary care, whereas buprenorphine is delivered through primary care settings, which have minimal ancillary services. Previous research has shown that retention in OMT is influenced by several in-treatment variables such as occurrence of depressive symptoms, social functioning [44] and trust in the physician [45]. Therefore, although more severely dependent patients were included in the methadone group, it is not entirely surprising that non-medical use of opioids did not differ from the buprenorphine group in our study because the methadone patients had access to more comprehensive care [46].

Some limitations of this study should be acknowledged. Data on substance use was based on self-reported behaviors, which are often questioned due to possible social desirability bias. However, the validity and reliability of self-reports about active drug use have been established in many studies that used similar methods for collecting information about substance use behaviors $[47,48]$ as well as in a previous study with a sample of HIV-infected patients in which substantial agreement was documented between self-reported heroin use and morphine detection in urine [49]. Our results do not allow a clear inference about the direction of effects. Although the study started some years ago, this cohort remains a very interesting observational sample of patients, especially for countries that have only recently started to scale up antiretrovirals and OMT for opioid dependent HIV-infected individuals. Finally, although a statistical adjustment was made to allow for non-random assignment to medication type, this study was not a controlled clinical trial and further investigations are required to confirm our findings.

\section{Conclusions}

The results of the present study contribute towards a better understanding of the factors associated with nonmedical use of opioids, and may also have implications for understanding adherence to anti-retroviral therapy in HIV-infected opioid-dependent individuals. When methadone and buprenorphine are effective in stabilizing opioid dependence, they can also improve health and socio-economic outcomes in HIV positive IDUs [50]. Clinically, these findings demonstrate the important relationship between withdrawal symptoms in OMT treated patients and positive response to medication. More generally, it emphasizes the importance of being attentive to the provided care and to the environment of patients. These findings corroborate the idea that stabilization on OMT is not only a patient problem [51]. Our data suggest that some combined and novel approaches are necessary to decrease the harms associated with abuse or dependence on benzodiazepines, cocaine, and/or cannabinoids, especially in HIV-infected individuals. Clinical intervention studies aimed at optimizing effectiveness of OMT in HIV-infected patients are required to better understand the extent of a more comprehensive approach combining attention to withdrawal symptoms, and treatment for other drug dependence. Programs with better patients retention can improve adherence to ART and HIV outcomes in people living with HIV. Further research on new medications and new interventions for co-dependence on stimulants and/or benzodiazepines is required to develop a more comprehensive and effective model of care for such populations and to assure improved OMT and HIV outcomes.

\section{List of Abbreviations}

ANRS: Agency for Research on Aids and Viral Hepatitis; ART: Antiretroviral Treatment; CD4: Cluster of Differentiation 4; CES-D: Center for Epidemiological Studies Depression Scale; CPP: Committee for the Protection of Persons; GEE: Generalized Estimating Equations; HCV: Hepatitis C Virus; HIV: Human Immunodeficiency Virus; IDUs: Intravenous Drug Users; IQR: Interquartile Range; OMT: Opioid Maintenance Treatment; OR: Odds Ratio; RNA: RiboNucleic Acid.

\section{Acknowledgements}

This research was supported by The French National Agency for Aids Research (ANRS, France), the charity organization ECS-SIDACTION (France), and the Departmental Council (Bouches-du-Rhône, France). We thank all members of the MANIF 2000 cohort study group: C. Boirot, A.D. Bouhnik, M. P. Carrieri, J.P. Cassuto, M Chesney, J. Cohen, P. Dellamonica, P. Dujardin, H. Gallais, J.A. Gastaut, G. Lepeu, C Marimoutou, D. Mechali, J.P. Moatti, J. Moreau, M. Negre, Y. Obadia, I. Poizot-Martin, C. Pradier, M. Preau, D. Rey, P. Roux, C. Rouzioux, A. Sobel, B. Spire, F. Tremolieres, V. Villes, E. Vincent and D. Vlahov. All authors declare that they have no conflicts of interest. We especially thank all physicians and nurses who were involved in the cohort and all patients living with HIV who took part in this study.

\section{Author details}

${ }^{1}$ INSERM, U912 (SE4S), 23 rue Stanislas Torrents, 13006 Marseille, France. ${ }^{2}$ Université Aix Marseille, IRD, UMR-S912, Marseille, France. ${ }^{3}$ ORS PACA, Observatoire Régional de la Santé Provence Alpes Côte d'Azur, Marseille, France. ${ }^{4}$ Hôpital La Conception, Service des Maladies Infectieuses, 147 boulevard Baille, 13005 Marseille, France. ${ }^{5}$ King's College London, 4 Windsor Walk, London, SE5 8BB, UK. 'Division on Substance Abuse, New York State Psychiatric Institute and Columbia University, NY, USA.

\section{Authors' contributions}

PMC, IR and BS were involved in the study concept and design as well as the acquisition of data. Statistical analyses and interpretation of data were performed by PMC, JC, SDC, MG, PR and BS. PR was principally involved in the drafting of the manuscript under the supervision of PMC and SDC. All authors read and approved the final manuscript.

\section{Competing interests}

Dr. Comer has received research grant funding from NIDA (DA09236, DA10909), as well as unrestricted educational grants from Reckitt Benckiser and Schering Plough to study the abuse liability of buprenorphine. The other authors who have taken part in this study declared that they do not have anything to disclose regarding funding or conflict of interest with respect to this manuscript.

Received: 4 May 2011 Accepted: 28 November 2011 Published: 28 November 2011

\section{References}

1. Bobrova N, Sarang A, Stuikyte R, Lezhentsev K: Obstacles in provision of anti-retroviral treatment to drug users in Central and Eastern Europe 
and Central Asia: a regional overview. Int J Drug Policy 2007, 18(4):313-318.

2. Krupitsky E, Zvartau E, Karandashova G, Horton NJ, Schoolwerth KR, Bryant K, Samet JH: The onset of HIV infection in the Leningrad region of Russia: a focus on drug and alcohol dependence. HIV Med 2004, 5(1):30-33.

3. van Beek I: Case study: accessible primary health care-a foundation to improve health outcomes for people who inject drugs. Int I Drug Policy 2007, 18(4):329-332.

4. Gossop M, Marsden J, Stewart D, Treacy S: Reduced injection risk and sexual risk behaviours after drug misuse treatment: results from the National Treatment Outcome Research Study. AIDS Care 2002, 14(1):77-93.

5. Emmanuelli J, Desenclos JC: Harm reduction interventions, behaviours and associated health outcomes in France, 1996-2003. Addiction 2005, 100(11):1690-1700.

6. Lawrinson P, Ali R, Buavirat A, Chiamwongpaet S, Dvoryak S, Habrat B, Jie S, Mardiati R, Mokri A, Moskalewicz J, Newcombe D, Poznyak V, Subata E, Uchtenhagen A, Utami DS, Vial R, Zhao C: Key findings from the WHO collaborative study on substitution therapy for opioid dependence and HIV/AIDS. Addiction 2008, 103(9):1484-1492.

7. Roux P, Carrieri MP, Villes V, Dellamonica P, Poizot-Martin I, Ravaux I, Spire B: The impact of methadone or buprenorphine treatment and ongoing injection on highly active antiretroviral therapy (HAART) adherence: evidence from the MANIF2000 cohort study. Addiction 2008, 103(11):1828-1836.

8. Mills EJ, Nachega JB, Bangsberg DR, Singh S, Rachlis B, Wu P, Wilson K, Buchan I, Gill CJ, Cooper C: Adherence to HAART: a systematic review of developed and developing nation patient-reported barriers and facilitators. PLoS Med 2006, 3(11):e438.

9. Malta M, Strathdee SA, Magnanini MM, Bastos Fl: Adherence to antiretroviral therapy for human immunodeficiency virus/acquired immune deficiency syndrome among drug users: a systematic review. Addiction 2008, 103(8):1242-1257.

10. Maggiolo F, Airoldi M, Kleinloog HD, Callegaro A, Ravasio V, Arici C, Bombana E, Suter F: Effect of adherence to HAART on virologic outcome and on the selection of resistance-conferring mutations in NNRTI- or PItreated patients. HIV Clin Trials 2007, 8(5):282-292.

11. Lucas GM, Cheever LW, Chaisson RE, Moore RD: Detrimental effects of continued illicit drug use on the treatment of HIV-1 infection. J Acquir Immune Defic Syndr 2001, 27(3):251-259.

12. Carrieri MP, Moatti JP, Vlahov D, Obadia Y, Reynaud-Maurupt C, Chesney M: Access to antiretroviral treatment among French HIV infected injection drug users: the influence of continued drug use. MANIF 2000 Study Group. J Epidemiol Community Health 1999, 53(1):4-8.

13. Bouhnik AD, Chesney M, Carrieri P, Gallais H, Moreau J, Moatti JP, Obadia Y, Spire B: Nonadherence among HIV-infected injecting drug users: the impact of social instability. J Acquir Immune Defic Syndr 2002, 31(Suppl 3): S149-153.

14. Carrieri MP, Rey D, Loundou A, Lepeu G, Sobel A, Obadia Y: Evaluation of buprenorphine maintenance treatment in a French cohort of HIVinfected injecting drug users. Drug Alcohol Depend 2003, 72(1):13-21.

15. Gallant JE, Somani J, Chaisson RE, Stanton D, Smith M, Quinn TC: Diagnostic accuracy of three clinical case definitions for advanced HIV disease. Aids 1992, 6(3):295-299.

16. Fuhrer R, Rouillon F, Lellouch J: Diagnostic reliability among French psychiatrists using DSM-III criteria. Acta Psychiatr Scand 1986, 73(1):12-16

17. Radloff $L$ : The CES-D scale: a self-report depression scale for research in the general population. Appl Psychol Measure 1977, 3:385-491.

18. Carrieri MP, Roux P, Cohen J, Ravaux I, Dellamonica P, Protopopescu C, Spire B, Rosenblum A: Self-reported side effects in buprenorphine and methadone patients receiving antiretroviral therapy: results from the MANIF 2000 cohort study. Addiction 2010, 105(12):2160-2168.

19. Price AM, Ilgen MA, Bohnert AS: Prevalence and correlates of nonmedical use of prescription opioids in patients seen in a residential drug and alcohol treatment program. J Subst Abuse Treat 2011, 41(2):208-214.

20. Young AM, Havens JR, Leukefeld CG: Route of administration for illicit prescription opioids: a comparison of rural and urban drug users. Harm Reduct J 2010, 7:24.

21. Winstock AR, Lea T: Diversion and injection of methadone and buprenorphine among clients in public opioid treatment clinics in New South Wales, Australia. Subst Use Misuse 2010, 45(1-2):240-252.
22. Roux P, Villes V, Blanche J, Bry D, Spire B, Feroni I, Carrieri MP: Buprenorphine in primary care: Risk factors for treatment injection and implications for clinical management. Drug Alcohol Depend 2008, 97(12):105-113.

23. Spire B, Lucas GM, Carrieri MP: Adherence to HIV treatment among IDUs and the role of opioid substitution treatment (OST). Int I Drug Policy 2007, 18(4):262-270.

24. Bouhnik AD, Carrieri MP, Rey D, Spire B, Gastaut JA, Gallais H, Obadia Y: Drug injection cessation among HIV-infected injecting drug users. Addict Behav 2004, 29(6):1189-1197.

25. Altice FL, Mostashari F, Friedland GH: Trust and the acceptance of and adherence to antiretroviral therapy. J Acquir Immune Defic Syndr 2001, 28(1):47-58.

26. Williamson A, Darke S, Ross J, Teesson M: The effect of persistence of cocaine use on 12-month outcomes for the treatment of heroin dependence. Drug Alcohol Depend 2006, 81(3):293-300,

27. Brands B, Blake J, Marsh DC, Sproule B, Jeyapalan R, Li S: The impact of benzodiazepine use on methadone maintenance treatment outcomes. J Addict Dis 2008, 27(3):37-48.

28. Parr JM, Kavanagh DJ, Cahill L, Mitchell G, Mc DYR: Effectiveness of current treatment approaches for benzodiazepine discontinuation: a metaanalysis. Addiction 2009, 104(1):13-24.

29. Eiroa-Orosa FJ, Haasen C, Verthein U, Dilg C, Schafer I, Reimer J: Benzodiazepine use among patients in heroin-assisted vs. methadone maintenance treatment: findings of the German randomized controlled trial. Drug Alcohol Depend 2010, 112(3):226-233.

30. Vidal-Trecan G, Varescon I, Nabet N, Boissonnas A: Intravenous use of prescribed sublingual buprenorphine tablets by drug users receiving maintenance therapy in France. Drug Alcohol Depend 2003, 69(2):175-181.

31. Peretti-Watel P, Spire B, Lert F, Obadia Y: Drug use patterns and adherence to treatment among HIV-positive patients: evidence from a large sample of French outpatients (ANRS-EN12-VESPA 2003). Drug Alcohol Depend 2006, 82(Suppl 1):S71-79.

32. Gossop M, Marsden J, Stewart D, Treacy S: Outcomes after methadone maintenance and methadone reduction treatments: two-year follow-up results from the National Treatment Outcome Research Study. Drug Alcohol Depend 2001, 62(3):255-264.

33. Bell J, Burrell T, Indig D, Gilmour S: Cycling in and out of treatment; participation in methadone treatment in NSW, 1990-2002. Drug Alcohol Depend 2006, 81(1):55-61.

34. Elkader AK, Brands B, Callaghan R, Sproule BA: Exploring the relationship between perceived inter-dose opioid withdrawal and patient characteristics in methadone maintenance treatment. Drug Alcohol Depend 2009, 105(3):209-214.

35. Donny EC, Brasser SM, Bigelow GE, Stitzer ML, Walsh SL: Methadone doses of $100 \mathrm{mg}$ or greater are more effective than lower doses at suppressing heroin self-administration in opioid-dependent volunteers. Addiction 2005, 100(10):1496-1509.

36. Lopatko OV, White JM, Huber A, Ling W: Opioid effects and opioid withdrawal during a $24 \mathrm{~h}$ dosing interval in patients maintained on buprenorphine. Drug Alcohol Depend 2003, 69(3):317-322.

37. de Wet C, Reed L, Glasper A, Moran P, Bearn J, Gossop M: Benzodiazepine co-dependence exacerbates the opiate withdrawal syndrome. Drug Alcohol Depend 2004, 76(1):31-35.

38. Backmund M, Schutz CG, Meyer K, Eichenlaub D, Soyka M: Alcohol consumption in heroin users, methadone-substituted and codeinesubstituted patients-frequency and correlates of use. Eur Addict Res 2003, 9(1):45-50

39. Mateu-Gelabert P, Sandoval M, Meylakhs P, Wendel T, Friedman SR: Strategies to avoid opiate withdrawal: implications for HCV and HIV risks. Int J Drug Policy 2010, 21(3):179-185.

40. WHO: Guidelines for the Psychosocially Assisted Pharmacological Treatment of Opioid Dependence 2009 [http://whqlibdoc.who.int/publications/2009/ 9789241547543_eng.pdf;].

41. McCance-Katz EF, Rainey PM, Friedland G, Jatlow P: The protease inhibitor lopinavir-ritonavir may produce opiate withdrawal in methadonemaintained patients. Clin Infect Dis 2003, 37(4):476-482.

42. Michel L, Giorgi R, Villes V, Poizot-Martin I, Dellamonica P, Spire B, Protopopescu C, Carrieri MP: Withdrawal symptoms as a predictor of mortality in patients HIV-infected through drug use and receiving highly 
active antiretroviral therapy (HAART). Drug Alcohol Depend 2009, 99(13):96-104.

43. Mattick RP, Kimber J, Breen C, Davoli M: Buprenorphine maintenance versus placebo or methadone maintenance for opioid dependence. Cochrane Database Syst Rev 2008, , 2: CD002207.

44. Gerra G, Borella F, Zaimovic A, Moi G, Bussandri M, Bubici C, Bertacca S: Buprenorphine versus methadone for opioid dependence: predictor variables for treatment outcome. Drug Alcohol Depend 2004, 75(1):37-45.

45. Guichard A, Lert F, Brodeur JM, Richard L: Buprenorphine substitution treatment in France: drug users' views of the doctor-user relationship. Soc Sci Med 2007, 64(12):2578-2593.

46. Magura S, Nwakeze PC, Demsky SY: Pre- and in-treatment predictors of retention in methadone treatment using survival analysis. Addiction 1998, 93(1):51-60,

47. Darke S: Self-report among injecting drug users: a review. Drug Alcohol Depend 1998, 51(3):253-263, discussion 267-258.

48. Weiss RD, Najavits LM, Greenfield SF, Soto JA, Shaw SR, Wyner D: Validity of substance use self-reports in dually diagnosed outpatients. Am J Psychiatry 1998, 155(1):127-128.

49. Messiah A, Loundou AD, Maslin V, Lacarelle B, Moatti JP: Physician recognition of active drug use in HIV-infected patients is lower than validity of patient's self-reported drug use. J Pain Symptom Manage 2001, 21(2):103-112.

50. Quaglio G, Lugoboni F, Pattaro C, Montanari L, Lechi A, Mezzelani P, Des Jarlais DC: Patients in long-term maintenance therapy for drug use in Italy: analysis of some parameters of social integration and serological status for infectious diseases in a cohort of 1091 patients. BMC Public Health 2006, 6:216.

51. Newman RG: Adherence to methadone treatment: Not just a patient problem. J Subst Abuse Treat 2009, 36(4):463.

doi:10.1186/1477-7517-8-31

Cite this article as: Roux et al:: Non-medical use of opioids among HIVinfected opioid dependent individuals on opioid maintenance treatment: the need for a more comprehensive approach. Harm Reduction Journal 2011 8:31.

\section{Submit your next manuscript to BioMed Central and take full advantage of:}

- Convenient online submission

- Thorough peer review

- No space constraints or color figure charges

- Immediate publication on acceptance

- Inclusion in PubMed, CAS, Scopus and Google Scholar

- Research which is freely available for redistribution

Submit your manuscript at www.biomedcentral.com/submit
C Biomed Central 